\title{
Identifying conserved protein complexes between species by constructing interolog networks
}

\author{
Phi-Vu Nguyen ${ }^{1}$, Sriganesh Srihari ${ }^{2}$, Hon Wai Leong ${ }^{1 *}$ \\ From Asia Pacific Bioinformatics Network (APBioNet) Twelfth International Conference on Bioinformatics \\ (InCoB2013) \\ Taicang, China. 20-22 September 2013
}

\begin{abstract}
Background: Protein complexes conserved across species indicate processes that are core to cellular machinery (e.g. cell-cycle or DNA damage-repair complexes conserved across human and yeast). While numerous computational methods have been devised to identify complexes from the protein interaction (PPI) networks of individual species, these are severely limited by noise and errors (false positives) in currently available datasets. Our analysis using human and yeast PPI networks revealed that these methods missed several important complexes including those conserved between the two species (e.g. the MLH1-MSH2-PMS2-PCNA mismatch-repair complex). Here, we note that much of the functionalities of yeast complexes have been conserved in human complexes not only through sequence conservation of proteins but also of critical functional domains. Therefore, integrating information of domain conservation might throw further light on conservation patterns between yeast and human complexes.

Results: We identify conserved complexes by constructing an interolog network (IN) leveraging on the functional conservation of proteins between species through domain conservation (from Ensembl) in addition to sequence similarity. We employ 'state-of-the-art' methods to cluster the interolog network, and map these clusters back to the original PPI networks to identify complexes conserved between the species. Evaluation of our IN-based approach (called COCIN) on human and yeast interaction data identifies several additional complexes (76\% recall) compared to direct complex detection from the original PINs (54\% recall). Our analysis revealed that the INconstruction removes several non-conserved interactions many of which are false positives, thereby improving complex prediction. In fact removing non-conserved interactions from the original PINs also resulted in higher number of conserved complexes, thereby validating our IN-based approach. These complexes included the mismatch repair complex, MLH1-MSH2-PMS2-PCNA, and other important ones namely, RNA polymerase-II, EIF3 and MCM complexes, all of which constitute core cellular processes known to be conserved across the two species.

Conclusions: Our method based on integrating domain conservation and sequence similarity to construct interolog networks helps to identify considerably more conserved complexes between the PPI networks from two species compared to direct complex prediction from the PPI networks. We observe from our experiments that protein complexes are not conserved from yeast to human in a straightforward way, that is, it is not the case that a yeast complex is a (proper) sub-set of a human complex with a few additional proteins present in the human complex. Instead complexes have evolved multifold with considerable re-organization of proteins and redistribution of their functions across complexes. This finding can have significant implications on attempts to extrapolate other kinds of relationships such as synthetic lethality from yeast to human, for example in the identification of novel cancer targets. Availability: http://www.comp.nus.edu.sg/ leonghw/COCIN/.
\end{abstract}

\footnotetext{
* Correspondence: leonghw@comp.nus.edu.sg

'Department of Computer Science, National University of Singapore,

Singapore 117590

Full list of author information is available at the end of the article
}

(C) 2013 Nguyen et al.; licensee BioMed Central Ltd. This is an open access article distributed under the terms of the Creative Commons Attribution License (http://creativecommons.org/licenses/by/2.0), which permits unrestricted use, distribution, and reproduction in any medium, provided the original work is properly cited. 


\section{Background}

Complexes of physically interacting proteins form fundamental units responsible for driving key biological processes within cells. Even in the simple model organism Saccharomyces cerevisae (budding yeast), these complexes are composed to several protein subunits that work in a coherent fashion to carry out cellular functions. Therefore a faithful reconstruction of the entire set of complexes (the 'complexosome') from the set of physical interactions (the 'interactome') is essential to understand their organisation and functions as well as their roles in diseases [1-4].

In spite of the significant progress in computational identification of protein complexes from protein interaction (PPI) networks over the last few years (see the surveys $[1,2])$, computational methods are severely limited by noise (false positives) and lack of sufficient interactions (e.g. membrane-protein interactions) in currently available PPI datasets, particularly from human, to be able to completely reconstruct the complexosome [1,2]. For example, several complexes involved in core cellular processes such as cell cycle and DNA damage response (DDR) are not present in a recent (2012) compendium of human protein complexes (http://human.med.utoronto.ca/) assembled solely by computational identification of complexes from high-throughput PPIs[5]; a web-search (as of Feb 2013) in this compendium for BRCA1 does not yield any complexes even though BRCA1 is known to participate in three fundamental complexes in DDR viz. BRCA1-A, BRCA1-B and BRCA1-C complexes [6-8]. A possible reason for missing these complexes is the lack of sufficient PPI data required for identifying them even using the best available algorithms. But, the authors of this compendium note that many human complexes appear to be ancient and slowly evolving - roughly a quarter of the predicted complexes overlapped with complexes from yeast and fly, with half of their subunits having clear orthologs [5]. Therefore, it is useful to devise effective computational methods that look for evidence from evolutionary conservation to complement PPI data to reconstruct the full set of complexes.

In the attempt to integrate evolutionary information with PPI networks, Kelley et al. [9] and Sharan et al. [10] devised methods to construct an orthology graph of conserved interactions from two species, which in their experiments were yeast (S. cerevisae) and bacteria (H. pylori), using a sequence homology-based (using BLAST E-score similarity) mapping of proteins between the species. Dense sub-graphs induced in this orthology graph represented putative complexes conserved between the two species. The complexes so-identified were involved in core cellular processes conserved between the two species - e.g. those in protein translation, DDR and nuclear transport. Van Dam and Snel (2008) [11] studied rewiring of protein complexes between yeast and human using high-throughput PPI datasets mapped onto known yeast and human complexes. From their experiments, they concluded that a majority of co-complexed protein pairs retained their interactions from yeast to human indicating that the evolutionary dynamics of complexes was not due to extensive PPI network rewiring within complexes but instead due to gain or loss of protein subunits from yeast to human. Hirsh and Sharan [12] developed a protein evolution-based model and employed it to identify conserved protein complexes between yeast and fly, while Zhenping et al. [13] used integer quadratic programming to align and identify conserved regions in molecular networks. Marsh et al. [14] integrated data on PPI and structure to understand mechanisms of protein conservation; they found that during evolution gene fusion events tend to optimize complex assembly by simplifying complex topologies, indicating genome-wide pathways of complex assembly.

\section{Integrating domain conservation}

Inspired from these works, here we devise a novel computational method to identify conserved complexes and apply it to yeast and human datasets. A crucial point we note on the conservation from yeast to human is that many cellular mechanisms, though conserved, have in fact evolved many-fold in complexity - for example, cell cycle and DDR. Consequently, while several proteins in these mechanisms are conserved by sequence similarity (e.g. RAD9 and hRAD9), there are others that are unique (non-conserved) to human (e.g. BRCA1); see Figure 1. These non-conserved proteins perform similar functions (e.g. cell cycle and DDR) as their conserved counterparts, but do not show high sequence similarity to any of the yeast proteins. A deeper examination reveals that these proteins in fact contain conserved functional domains for example, the BRCT domain which is present in yeast RAD9 and human hRAD9 is also present in the nonconserved human BRCA1 and 53BP1; all of these play crucial roles in DDR [15]. Similar structure can be seen in the case of RecQ helicases - several helicase domains are conserved from the yeast SGS1 to human BLM and WRN, but there are three helicases RECQ1,4,5 which are unique to human that also contain these helicase domains [16]. Therefore, integrating information on functional conservation, mainly through domain conservation, can help to identify considerably more (functionally) conserved complexes than mere sequence similarity, thereby throwing further light on the conservation patterns of complexes in particular and cellular processes in general.

In order to achieve this, simple BLAST-based scores as used in earlier works [9-13] to measure homology between yeast and human proteins do not suffice. Here, we integrate multiple databases including Ensembl [17] and OrthoMCL [18] to build homology relationships 


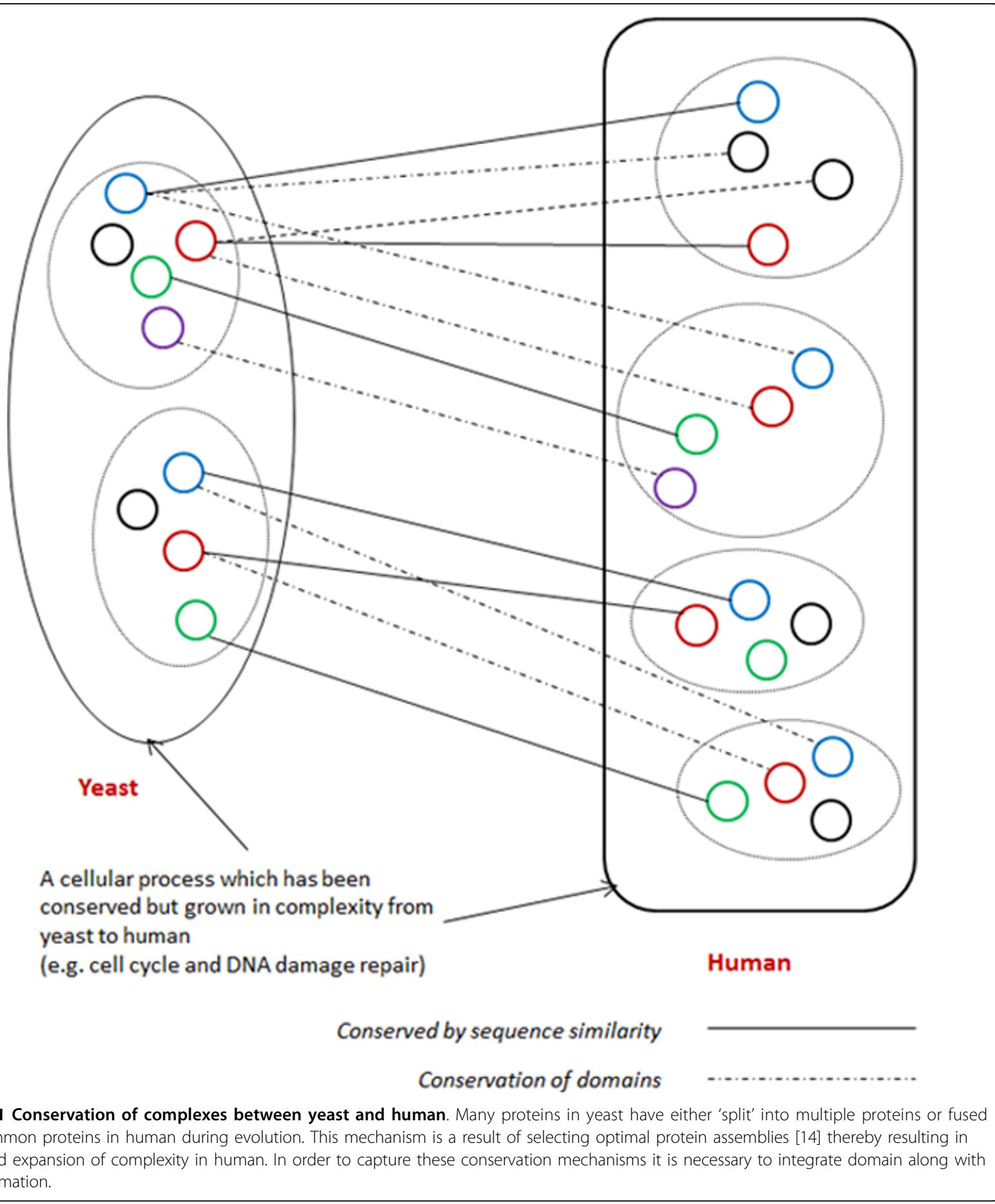

among proteins; these databases use a variety of information to construct orthologous groups among proteins including checking for conserved domains. The integration of these databases generates many-to-many correspondence between yeast and human proteins instead of the predominantly one-to-one correspondence obtained by from BLAST-based similarity.
We devise a novel computational method to construct an interolog network using domain information along with PPI conservation between human and yeast. Next, we identify dense clusters within the interolog network using current 'state-of-the-art' PPI-clustering methods (as against traditional clustering methods used in $[9,10])$. These clusters when mapped back to the PPI 
networks reveal conserved dense regions, many of which correspond to conserved complexes.

Our experiments here reveal that,

(i) integrating domain information generates many valuable interactions from the many-to-many ortholog relationships in the interolog network, thereby enhancing its quality;

(ii) interolog network also reduces false-positive interactions by accounting for conserved PPIs;

(iii) our interolog network construction aids clustering algorithms to identify far more conserved complexes than direct clustering of the individual PPI networks; and

(iv) many of these conserved complexes are involved in core cellular processes such as cell cycle and DDR throwing further light to the conservation of these cellular processes.

We call our method COCIN (COnserved Complexes from Interolog Networks).

\section{Methods}

\section{Constructing the interolog network}

Given two PPI networks from two species $S_{1}$ and $S_{2}$, and the homology information between proteins of the two networks, we construct an interolog network $\mathrm{G}_{\mathrm{I}}$ as follows. The two PPI networks are represented as $G_{1}$ $\left(V_{1}, E_{1}\right)$ and $G_{2}\left(V_{2}, E_{2}\right)$, and the homology relationship between the proteins is governed by a many-to-many correspondence $\theta: \mathrm{V}_{1} \rightarrow \mathrm{V}_{2}$. The interolog network is defined as $G_{I}\left(V_{I}, E_{I}\right)$, where $V_{I}=\left\{v_{I}=\{p, q\} \mid p \in V_{1}\right.$, $\mathrm{q} \in \mathrm{V}_{2}$, and $\left.(\mathrm{p}, \mathrm{q}) \in \theta\right\}$, and $\mathrm{E}_{\mathrm{I}}=\left\{\left(\mathrm{v}_{\mathrm{I}}, \mathrm{v}_{\mathrm{I}}^{\prime}\right) \mid \mathrm{v}_{\mathrm{I}}=\{\mathrm{p}, \mathrm{q}\} ; \mathrm{v}_{\mathrm{I}=}^{\prime}\{\mathrm{r}\right.$, $\mathrm{s}\} ;(\mathrm{p}, \mathrm{r}) \in \mathrm{E}_{1}$ and $\left.(\mathrm{q}, \mathrm{s}) \in \mathrm{E}_{2}\right\}$.
Each node in the interolog network represents a pair of homologous proteins, one from each species. Each edge in the interolog network represents an interaction that is conserved in both species (interolog). However, if a protein $\mathrm{p} \in \mathrm{V}_{1}$ can be orthologous to multiple proteins $x \in V_{2}$ and $x \in V_{2}$, then we add two vertices to $G_{I}$ namely $\{\mathrm{p}, \mathrm{x}\}$ and $\{\mathrm{p}, \mathrm{y}\}$, and add an edge between two vertices. Doing so integrates the many-to-many relationships obtained due to domain conservation into the interolog network. Figure 2 below gives a simple example of this network-construction.

Any connected sub-network in this interolog network can be mapped back to conserved sub-networks in the two PPI networks, and this is similar to the orthology graph method introduced by Kelley et al. [9] and Sharan et al. [10]. However, one unique advantage of our interolog network offers is that we can infer a collection of homologous complexes between the species. This property is highly relevant for identifying conserved complexes between yeast and human (revisit Figure 1).

In order to achieve this, we integrate multiple databases including Ensembl [17] and OrthoMCL [18] to build our homology relationships among proteins; these databases use a variety of information to construct orthologous groups among proteins including checking for conserved domains.

\section{Clustering the interolog network and detection of conserved complexes}

We identify dense clusters in the interolog network to detect conserved complexes between the two species. To do this, we tested a variety 'state-of-the-art' PPI network-clustering methods, and found the following three

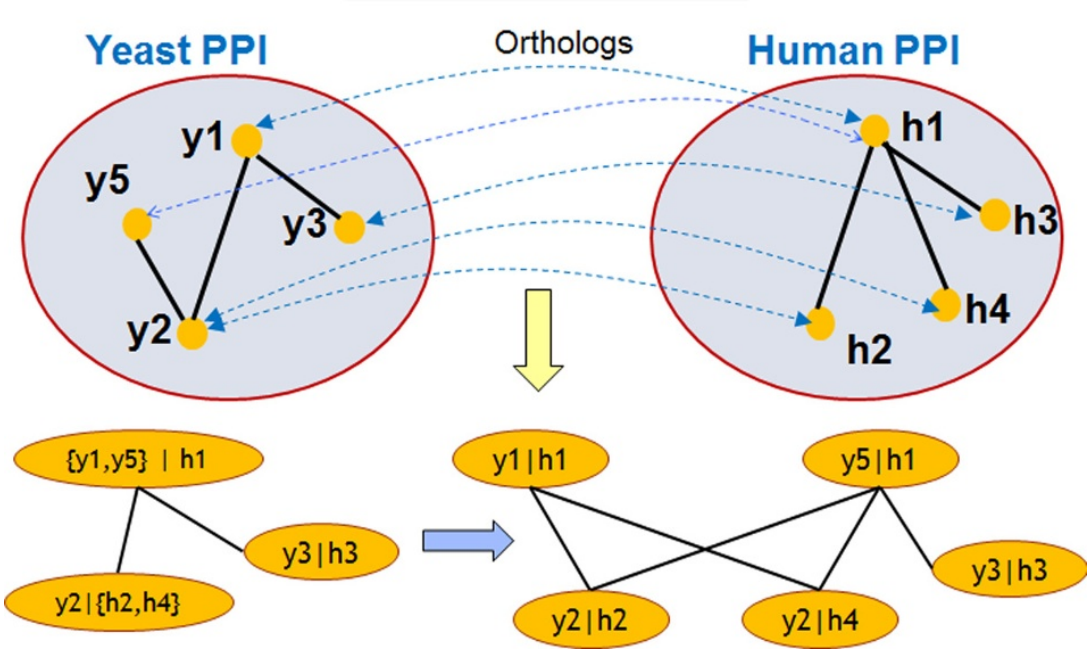

Figure 2 Construction of the interolog network - a simplified example. Our interolog network constructing integrates PPI and domain conservation information to generate a network that is conducive for clustering algorithms to identify considerably more conserved complexes compared to direct clustering of the original PPI networks from species. 
to perform the best - CMC (Clustering by merging Maximal Cliques) by Liu et al. [19], MCL (Markov Clustering) by van Dongen [20] and HACO (Hierarchical Clustering with Overlaps) by Wang et al. [21]. The comparative assessment of these methods has been confirmed with earlier works [1,2,22-24].

$\mathrm{CMC}$ operates by first enumerating all maximal cliques in network, and ranks them in descending order of the weighted interaction density. It then iteratively merges highly overlapping cliques to identify dense clusters in the network. MCL simulates a series of random paths (called a flow) and iteratively decomposes the network into a number of dense clusters. HACO performs hierarchical clustering by repeatedly identifying smaller dense clusters and merging these into larger clusters. HACO has an advantage over the traditional hierarchical clustering because it allows for overlaps (protein-sharing) among the clusters.

Upon finding dense clusters in the interolog network, we map back these clusters to sub-networks within the two PPI networks to identify conserved complexes.

\section{Building a benchmark dataset for conserved protein complexes}

Due to lack of benchmark datasets of conserved protein complexes between human and yeast in the literature, we built our own "gold standard" conserved dataset as follows. Using currently available datasets of manually curated protein complexes of human and yeast, we selected pairs of complexes that shared significant fraction of (homologous) proteins.
For measuring the conservation level of a given complex pair $\left\{C_{1}, C_{2}\right\}$, where $C_{1}$ belongs to species $S_{1}$ and $C_{2}$ belongs to species $\mathrm{S}_{2}$, we adopted the following Multi-set Jaccard score:

Multi-set Jaccard score: Let $\mathrm{G}_{\mathrm{C} 1}$ and $\mathrm{G}_{\mathrm{C} 2}$ be the collections of ortholog groups in complexes $C_{1}$ and $C_{2}$, respectively. For any group $g_{i} \in G c_{i}(i=1,2)$, let $I_{C i}$ represent the multiplicity of the group $g_{i}$ in complex $C_{i}$, which essentially is the number of paralogs within the group. Multi-set Jaccard score is then given by:

$$
\operatorname{MSJ}\left(C_{1}, C_{2}\right)=\frac{\sum_{g_{i} \in\left(G_{C 1} \cup G_{C 2}\right)} \min \left(\mathrm{I}_{C_{1}}\left(g_{i}\right), \mathrm{I}_{C_{2}}\left(g_{i}\right)\right)}{\sum_{g_{i} \in\left(G_{C 1} \cup G_{C 2}\right)} \max \left(\mathrm{I}_{C_{1}}\left(g_{i}\right), \mathrm{I}_{C_{2}}\left(g_{i}\right)\right)},
$$

There are often duplication of genes (paralogs) within complexes and clusters. Therefore, MSJ takes into account the multiplicity of the groups and does a more conservative and accurate estimation of the conservation between $C_{1}$ and $C_{2}$. See Figure 3 for an illustration.

We selected pairs of complexes that show MSJ $\geq 50 \%$ (see result section for details).

\section{Results}

\section{Preparation of experimental data}

We combined multiple PPI datasets to enhance the coverage of our interactome. We collected PPIs from IntAct [25] (version November 13, 2012) and Biogrid [26] (versions 3.2.95 and 3.2.89) databases for yeast; and from

\section{- Conservation scores:}

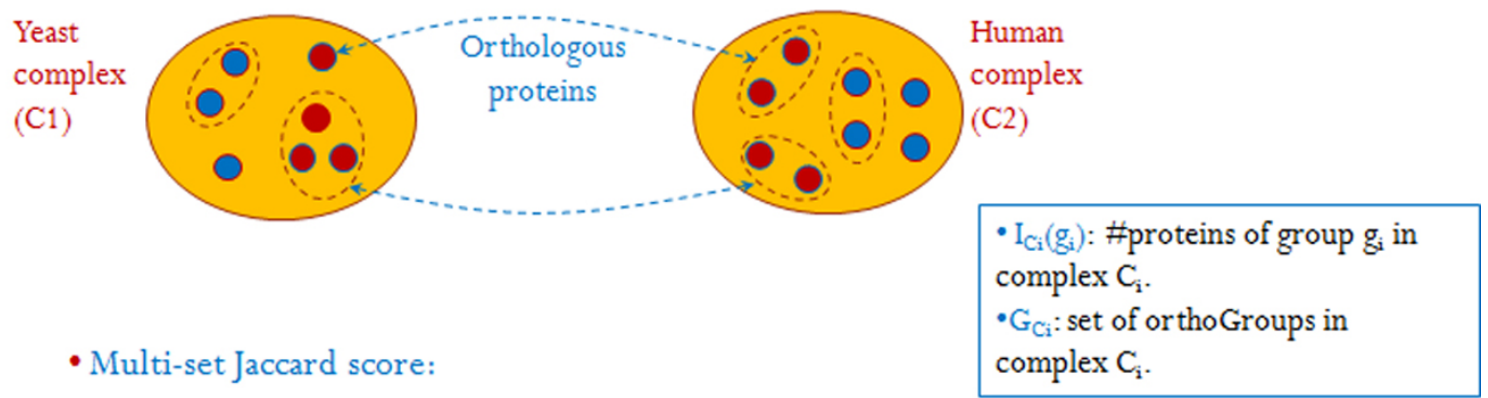

$$
\begin{gathered}
\operatorname{MSJ}\left(\mathrm{C}_{1}, \mathrm{C}_{2}\right)=\frac{\sum_{g_{i} \in\left(G_{c 1} \cup\left(G_{c 1} \cup G_{c 2}\right)\right.} \min \left(\mathrm{I}_{C 1}\left(g_{i}\right), \mathrm{I}_{C 2}\left(g_{i}\right)\right)}{\max \left(\mathrm{I}_{C 1}\left(g_{i}\right), \mathrm{I}_{C 2}\left(g_{i}\right)\right)}=\frac{\min (1,2)+\min (3,2)}{7+\max (1,2)+\max (3,2)}=0.25 \\
\cdot 0 \leq \mathrm{MSJ} \leq 1\left(\forall \mathrm{C}_{1}, \mathrm{C}_{2}\right)
\end{gathered}
$$

Figure 3 Conservation scores for building benchmark complex datasets. We generate a "gold standard" conserved complexes dataset to test our method. We use two scores here - the Jaccard score for orthologous groups and multi-set Jaccard score. 
Table 1 Properties of yeast physical PPI datasets

\begin{tabular}{lll}
\hline Database & $\begin{array}{l}\# \\
\text { proteins }\end{array}$ & $\begin{array}{l}\text { \# (non self and duplicated) } \\
\text { interactions }\end{array}$ \\
\hline $\begin{array}{l}\text { IntAct (version Nov 13, } \\
\text { 2012) }\end{array}$ & 5276 & 18834 \\
\hline $\begin{array}{l}\text { Biogrid (version 3.2.95, Nov } \\
\text { 30, 2012) }\end{array}$ & 5886 & 73923 \\
\hline IntAct UBiogrid & 6332 & 83777 \\
\hline IntActnBiogrid & 4620 & 8930 \\
\hline ICDScore(IntAct U Biogrid) & 5239 & 71636 \\
\hline
\end{tabular}

Biogrid [26] and HPRD [27] (Release 9, 2010) for human. Table 1 and 2 summarise these datasets.

Yeast curated complexes were gathered from Wodak database (CYC2008) [28] and human curated complexes from CORUM (version 09/2009) [29]; these form our benchmark complex datasets (details in Table 3). We used Ensembl [17] and OrthoMCL [18] for the homology mapping between human and yeast proteins.

\section{Criteria for evaluating predicted complexes}

For a predicted complex $C_{i}$ of one species and a manually curated (benchmark) complex $B_{j}$, we used Jaccard score based on collections of complex proteins: $J\left(C_{i}, B_{j}\right)=\frac{\left|C_{i} \cap B_{j}\right|}{\left|C_{i} \cup B_{j}\right|}$, which considers $C_{i}$ a correct prediction for $B_{j}$ if $J\left(C_{i}, B_{j}\right) \geq t$, a match threshold. We chose $t=$ 0.50 in our experiments as suggested by earlier works $[19,22] . C_{i}$ is then referred to as a matched prediction or matched predicted complex, and $B_{j}$ is referred to as a derived benchmark complex.

Based on this, precision is computed as the fraction of predicted complexes matching benchmark complexes, and the recall is computed as the fraction of benchmark protein complexes covered by our predicted complexes. A correctly predicted complex is also checked against our "gold standard" testing dataset to see if it is a conserved complex, in which case the derived complex is a derived conserved complex.

Results of complex detection using interolog network (IN) Table 4 summarizes the interolog network constructed from yeast and human PPIs. We map back each predicted

Table 2 Properties of human physical PPI datasets

\begin{tabular}{|c|c|c|}
\hline Database & \# proteins & \#interactions \\
\hline HPRD (Release 9, 2010) & 9617 & 39184 \\
\hline Biogrid (April 25, 2012) & 12515 & 59027 \\
\hline HPRD U Biogrid & 13624 & 76719 \\
\hline HPRD $\cap$ Biogrid & 8615 & 21491 \\
\hline ICDScore(HPRD U Biogrid) & 8521(EntrezID) & 61868 \\
\hline ICDEnrich(HPRD U Biogrid) & 9764 (EntrezlD) & 192053 (EntrezID) \\
\hline
\end{tabular}

Table 3 Properties of manually curated protein complex datasets

\begin{tabular}{ll}
\hline Databases & \# complexes \\
\hline Wodak[28] yeast complexes (CYC 2008) & $\mathbf{1 4 9}$ with size>3 \\
\cline { 2 - 2 } & $\mathbf{( 3 6 . 5 \% )}$ \\
\hline Cotal: 408 \\
\hline 2009) & $\mathbf{7 2 2}$ with size>3 (39.1\%) \\
\cline { 2 - 2 } & Total: 1843 \\
\hline
\end{tabular}

cluster from the IN to the original PPI networks to predict conserved complexes between the two species.

Firstly, we compared the results of complex detection from COCIN with direct clustering of the original PPI networks using CMC, HACO and MCL as shown in Tables 5 and 6. Interestingly, we observed that COCIN, which employs $\mathrm{CMC}, \mathrm{HACO}$ and $\mathrm{MCL}$ for clustering the interolog network, yielded a better recall than these methods on the original PPI networks. Further, because IN capitalises on the existence of interactions in both PPI networks (that is, conservation of interactions), the number of noisy dense clusters in COCIN is considerably reduced thereby enhancing its precision.

Figure 4 compares a predicted complex $C_{i}$ through COCIN with two predictions $C_{y}$ and $C_{h}$ from the original PPI networks; $C_{y}$ and $C_{h}$ form a pair of orthologous complexes, but by direct clustering of the original PPI networks and matching them and not using COCIN. We noticed that $C_{y}$ and $C_{h}$ contained several noisy proteins and interactions among them which were false positives. These false positives reduced the Jaccard accuracy of these complexes when matched to known benchmark complexes. We also note that when we computed the complex-derivability index called Component-Edge score (this index measures how much of chance a complex can be detected given the topology of a PPI network) proposed in [24], $C_{i}$ had a higher CE-score compared to $C_{y}$ and $C_{h}$ in the networks.

Figure 5 highlights the improvement of COCIN over $\mathrm{CMC}$, that is, the additional protein complexes of human and yeast detected by COCIN. As many noisy interactions are removed in the IN, among the conserved complexes that are detected by both CMC and COCIN, COCIN on an average obtained higher Jaccard scores. Some important additional conserved complexes found

Table 4 Properties of the interolog network constructed
from yeast and human PPIs
\begin{tabular}{ll}
\hline \# Mapped nodes using orthology & 2470 \\
\hline \# Interologs & 6133 \\
\hline Size of biggest connected component & 2434 nodes, 6112 edges \\
\hline \#Other connected components & 16 (size from 2-3) \\
\hline
\end{tabular}


Table 5 Comparisons of different methods on yeast data.

\begin{tabular}{lllllll}
\hline Method & $\begin{array}{l}\text { \# Predicted } \\
\text { complexes }\end{array}$ & $\begin{array}{l}\text { \# Matched } \\
\text { predictions }\end{array}$ & Precision & $\begin{array}{l}\text { \# Gold standard conserved } \\
\text { complexes }\end{array}$ & $\begin{array}{l}\text { \# Detected conserved } \\
\text { complexes }\end{array}$ & $\begin{array}{l}\text { Recall (of conserved } \\
\text { complexes) }\end{array}$ \\
\hline COCIN & 71 & 36 & $\mathbf{5 0 . 7 \%}$ & 42 & 32 & $\mathbf{7 6 . 2 \%}$ \\
\hline CMC & 1202 & 145 & $12.1 \%$ & 42 & 23 & $54.8 \%$ \\
\hline HACO & 1040 & 69 & $6.6 \%$ & 42 & 17 & $40.5 \%$ \\
\hline MCL & 387 & 37 & $9.6 \%$ & 42 & 5 & $11.9 \%$ \\
\hline
\end{tabular}

Predicted complexes: resulting network clusters

Matched predictions: resulting network clusters that match with benchmarks

Precision $=$ \#matched prediction/\#predicted complexes

Recall = \# detected conserved complexes/\# gold standard conserved complexes

Table 6 Comparisons of different methods on human data

\begin{tabular}{lllllll}
\hline $\begin{array}{l}\text { Method } \\
\text { complexes }\end{array}$ & $\begin{array}{l}\text { \# Matched } \\
\text { predictions }\end{array}$ & Precision & $\begin{array}{l}\text { \# Gold standard conserved } \\
\text { complexes }\end{array}$ & $\begin{array}{l}\text { \# Detected conserved } \\
\text { complexes }\end{array}$ & $\begin{array}{l}\text { Recall (of conserved } \\
\text { complexes) }\end{array}$ \\
\hline COCIN & 71 & 36 & $\mathbf{5 0 . 7 \%}$ & 118 & 78 & $\mathbf{6 6 . 1 \%}$ \\
\hline CMC & 1389 & 156 & $11.2 \%$ & 118 & 66 & $55.9 \%$ \\
\hline HACO & 1290 & 80 & $6.2 \%$ & 118 & 36 & $30.5 \%$ \\
\hline MCL & 631 & 45 & $7.1 \%$ & 118 & 24 & $20.3 \%$ \\
\hline
\end{tabular}

Predicted complexes: resulting network clusters

Matched predictions: resulting network clusters that match with benchmarks

Precision $=$ \#matched prediction/\#predicted complexes

Recall = \# detected conserved complexes/\# gold standard conserved complexes

One predicted complex of COCIN can match with many benchmark complexes, this explains for \#detected conserved complexes > \#matched predictions (as illustrated in Figures 5-8

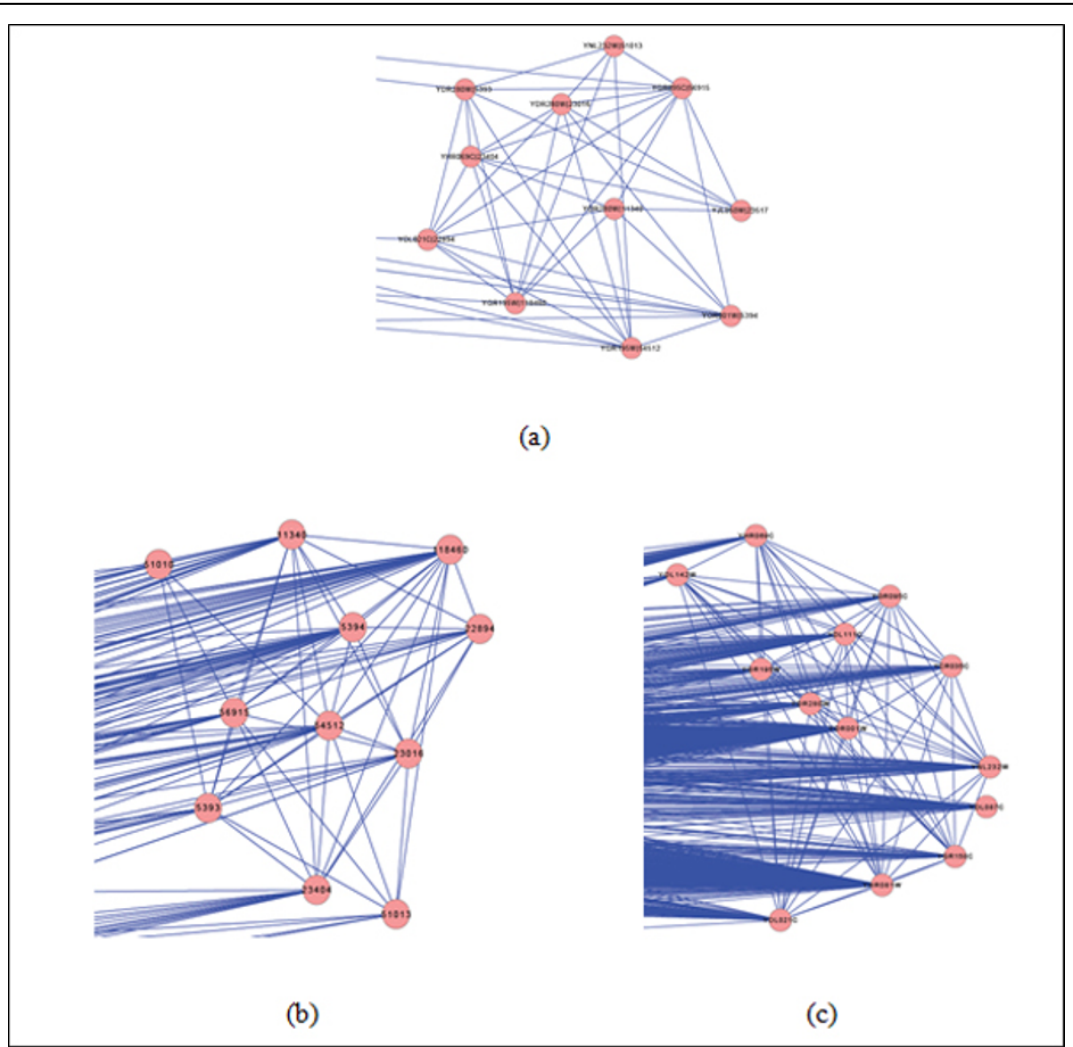

Figure 4 An illustration on a predicted complexes from IN. (a) A predicted complex in the IN. (b) The corresponding complex in the human PPI network. (c) The corresponding complex in the yeast PPI network. 


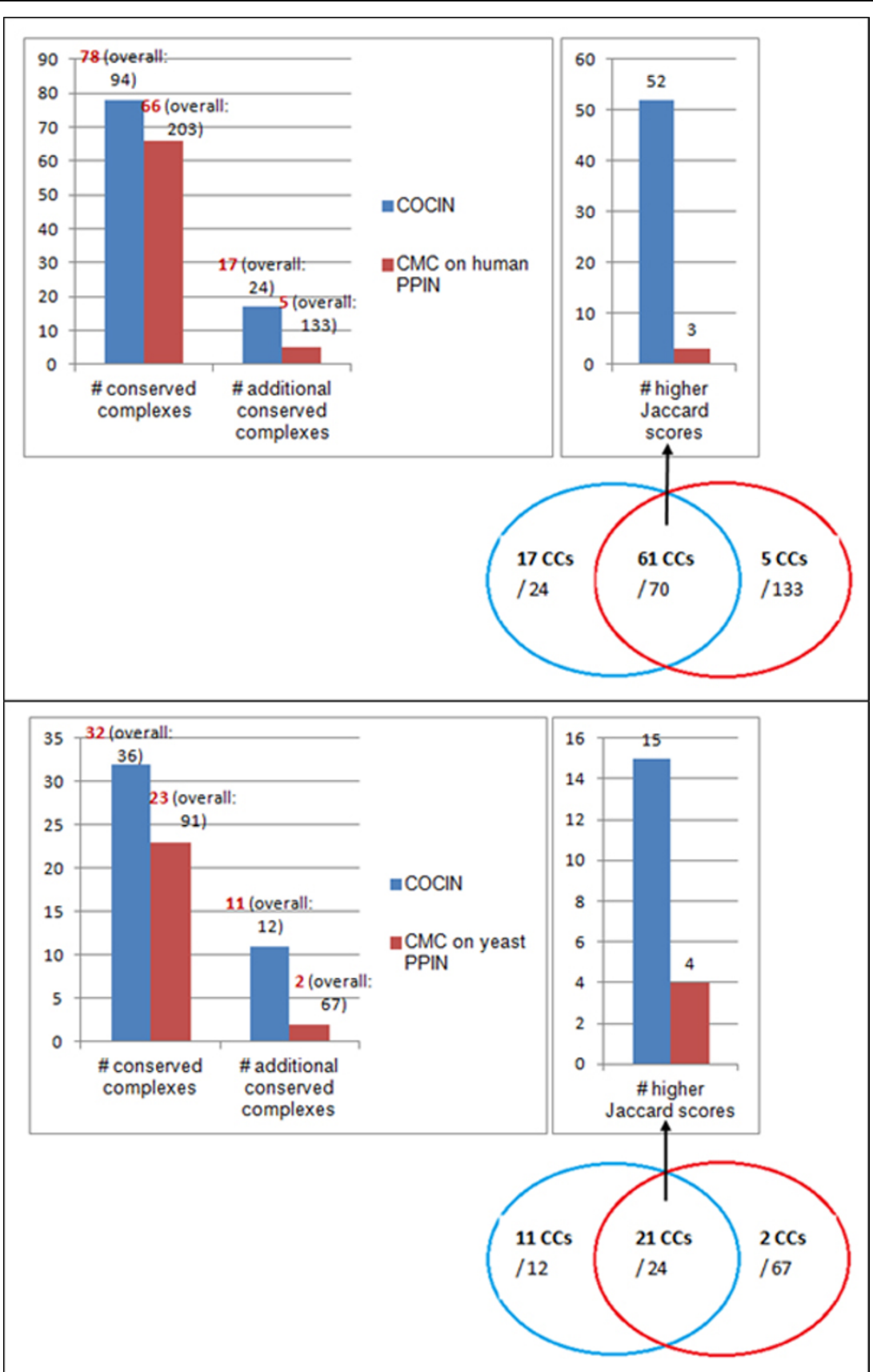

Figure 5 COCIN compared to CMC. COCIN over the interolog network identifies significantly more conserved complexes compared to direct clustering of the original PPI networks using CMC [19].

using COCIN were: RNA Polymerase II, EIF3 complex, MSH2-MLH1-PMS2-PCNA DNA-repair initiation complex, MCM complex, MMR complex, Ubiquitin E3 ligase, transcription factor TFIID, DNA replication factor C, $20 \mathrm{~S}$ proteasomes (descriptions of these complexes are listed in Tables 7 and 8).

\section{The result of complex detection in the conserved} subnetworks

To further understand the advantage of COCIN on leveraging conservation for better detection of complexes, we performed another experiment alternative to the interolog network as follows. We predicted complexes from the 
Table 7 Additional conserved complexes found in yeast

\begin{tabular}{|c|c|c|c|c|c|}
\hline ID & Complex name & Size & $\begin{array}{l}\text { Jaccard } \\
\text { score }\end{array}$ & $\begin{array}{l}\text { Functional } \\
\text { category }\end{array}$ & Functional description \\
\hline 96 & elF3 complex & 7 & 0.63 & Translation & Eukaryotic translation initiation factor \\
\hline 247 & $\begin{array}{l}\text { Transcription factor } \\
\text { TFIID complex }\end{array}$ & 15 & 0.73 & Transcription & mRNA synthesis \\
\hline 27 & $\begin{array}{l}\text { DNA-directed RNA } \\
\text { polymerase II complex }\end{array}$ & 12 & 0.69 & Transcription & mRNA synthesis \\
\hline 45 & $\begin{array}{l}\text { DNA replication factor } \\
\text { C complex (Rad24p) }\end{array}$ & 5 & 0.67 & DNA processing & DNA synthesis and replication \\
\hline 152 & $\begin{array}{l}\text { DNA replication factor } \\
\text { C complex (Rcf1p) }\end{array}$ & 5 & 0.67 & DNA processing & DNA synthesis and replication \\
\hline 294 & Mcm2-7 complex & 6 & 0.6 & DNA processing & Chromosome maintainance, DNA synthesis and replication \\
\hline 268 & SF3b complex & 6 & 0.57 & RNA processing & mRNA splicing \\
\hline 65 & U6 snRNP complex & 8 & 0.5 & RNA processing & $\begin{array}{l}\text { This complex combines with other snRNPs, unmodified pre-mRNA, and various } \\
\text { other proteins to assemble a spliceosome, a large RNA-protein molecular } \\
\text { complex upon which splicing of pre-mRNA occurs. }\end{array}$ \\
\hline 375 & AP-3 adaptor complex & 4 & 0.67 & $\begin{array}{l}\text { Cellular transport, } \\
\text { vesicular transport }\end{array}$ & $\begin{array}{l}\text { This complex is responsible for protein trafficking to lysosomes and other } \\
\text { related organelles. }\end{array}$ \\
\hline 25 & $20 S$ proteasome & 14 & 0.5 & $\begin{array}{l}\text { Cell cycle, protein } \\
\text { fate }\end{array}$ & $\begin{array}{l}\text { Proteasomal degradation (ubiquitin/proteasomal pathway), protein processing } \\
\text { (proteolytic) }\end{array}$ \\
\hline 137 & $\begin{array}{l}\text { Chaperonin-containing } \\
\text { T-complex }\end{array}$ & 8 & 0.67 & Protein fate & $\begin{array}{l}\text { A multisubunit ring-shaped complex that mediates protein folding in the } \\
\text { cytosol without a cofactor. }\end{array}$ \\
\hline
\end{tabular}

Table 8 Additional conserved complexes found in human

\begin{tabular}{|c|c|c|c|c|c|}
\hline ID & Complex name & Size & $\begin{array}{l}\text { Jaccard } \\
\text { score }\end{array}$ & $\begin{array}{l}\text { Functional } \\
\text { category }\end{array}$ & Function description \\
\hline 4392 & $\begin{array}{l}\text { EIF3 complex (EIF3A, EIF3B, } \\
\text { EIF3G, EIF3I, EIF3C) }\end{array}$ & 5 & 0.57 & Translation & Translation initiation \\
\hline 4403 & $\begin{array}{l}\text { EIF3 complex (EIF3A, EIF3B, } \\
\text { EIF3G, EIF3I, EIF3J) }\end{array}$ & 5 & 0.57 & Translation & Translation initiation \\
\hline 104 & RNA polymerase II core complex & 12 & 0.69 & Transcription & mRNA synthesis \\
\hline 2685 & RNA polymerase II & 17 & 0.59 & Transcription & mRNA synthesis \\
\hline 2686 & $\begin{array}{l}\text { BRCA1-core RNA polymerase II } \\
\text { complex }\end{array}$ & 13 & 0.64 & Transcription & mRNA synthesis \\
\hline 471 & PCAF complex & 10 & 0.6 & $\begin{array}{l}\text { Transcription, DNA } \\
\text { processing }\end{array}$ & $\begin{array}{l}\text { DNA conformation modification (e.g. chromatin), modification by } \\
\text { acetylation, deacetylation, organization of chromosome structure. }\end{array}$ \\
\hline 2200 & RFC2-5 subcomplex & 4 & 0.5 & DNA processing & DNA synthesis and replication \\
\hline 387 & MCM complex & 6 & 0.6 & DNA processing & Chromosome maintainance, DNA synthesis and replication \\
\hline 369 & MMR complex 2 & 4 & 0.67 & DNA processing & DNA damage repair \\
\hline 290 & $\begin{array}{l}\text { MSH2-MLH1-PMS2-PCNA DNA- } \\
\text { repair initiation complex }\end{array}$ & 4 & 0.67 & DNA processing & DNA damage repair initiation \\
\hline 1169 & SNARE complex & 4 & 0.6 & $\begin{array}{l}\text { Cellular transport, } \\
\text { vesicular transport }\end{array}$ & Vesicle fusion, synaptic vesicle exocytosis \\
\hline 562 & LSm2-8 complex & 7 & 0.67 & RNA processing & mRNA splicing \\
\hline 561 & LSm1-7 complex & 7 & 0.67 & RNA processing & Control of mRNA stability during splicing \\
\hline 3036 & $\begin{array}{l}\text { Ubiquitin E3 ligase (SKP1A, SKP2, } \\
\text { CUL1, CKS1B, RBX1) }\end{array}$ & 5 & 0.5 & $\begin{array}{l}\text { Cell cycle, protein } \\
\text { fate }\end{array}$ & $\begin{array}{l}\text { Mitotic cell cycle and cell cycle control, modification by } \\
\text { ubiquitination, deubiquitination }\end{array}$ \\
\hline 2188 & $\begin{array}{l}\text { Ubiquitin E3 ligase (CDC34, } \\
\text { NEDD8, BTRC, CUL1, SKP1A, } \\
\text { RBX1) }\end{array}$ & 5 & 0.5 & $\begin{array}{l}\text { Cell cycle, protein } \\
\text { fate }\end{array}$ & $\begin{array}{l}\text { Mitotic cell cycle and cell cycle control, modification by } \\
\text { ubiquitination, deubiquitination }\end{array}$ \\
\hline 2189 & $\begin{array}{l}\text { Ubiquitin E3 ligase (SMAD3, } \\
\text { BTRC, CUL1, SKP1A, RBX1) }\end{array}$ & 5 & 0.5 & $\begin{array}{l}\text { Cell cycle, protein } \\
\text { fate }\end{array}$ & $\begin{array}{l}\text { Mitotic cell cycle and cell cycle control, modification by } \\
\text { ubiquitination, deubiquitination }\end{array}$ \\
\hline
\end{tabular}




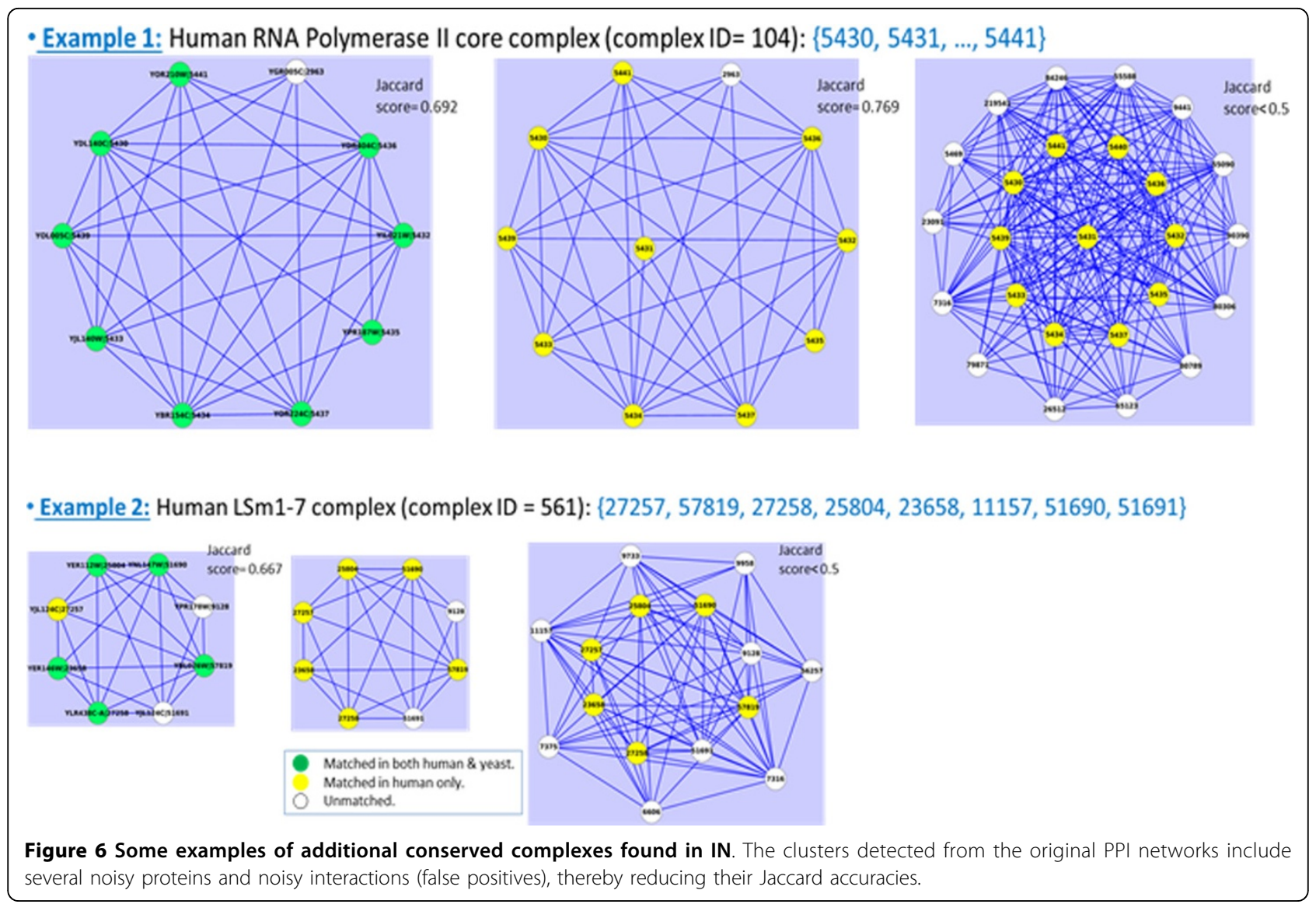

subset of protein interactions of the first species that are conserved in the second (we call this the conserved subnetwork in the first species). However, this can only find complexes of one species at a time, so we map these predicted complexes onto the PPI network of the other species to identify the corresponding conserved complexes. We employed CMC to do clustering on the conserved subnetworks.

Complex prediction from conserved subnetworks showed similar result as COCIN - 16 additional conserved complexes in human and 9 additional conserved complexes in yeast are found. This supported the purpose of IN - to leverage conserved interactions for improving complex prediction.

Figure 6 shows two other examples that explain why additional conserved complexes are found by COCIN but missed by CMC. We see from this picture that the predicted human complex from IN (the leftmost figure) and the corresponding predicted complex from the conserved subnetwork (the center figure) were contained in a larger CMC-predicted complex (the rightmost figure) from the original PPI networks. This larger complex included several noisy proteins that reduce the accuracy of the complex, thereby causing the complex to be missed.

\section{Comparisons with other complex detection methods in PPI networks}

Similar results were obtained using the other two methods HACO and MCL as well, thereby supporting the effectiveness of COCIN in identifying conserved protein complexes. Tables 5 and 6 present these comparisons in more details, while Figures 7 and 8 highlight further substantiate these results.

\section{Integrating domain information significantly enhances interolog construction}

Finally, Table 9 summarizes the quality of our testing dataset for conserved protein complexes between yeast and human. We compared the number of benchmark conserved complexes found in both human and yeast using mappings from Ensembl and OrthoMCL under multiple conservation score thresholds (Figure 9). Note that Ensembl contains homology information based on both sequence similarity as well as domain conservation, while OrthoMCL is predominantly based on sequence similarity. We noticed that using Ensembl homology information can yield more conserved complexes at all conservation score thresholds. Further, Figure 10 shows that there exist 1-to-many and many-to-many 


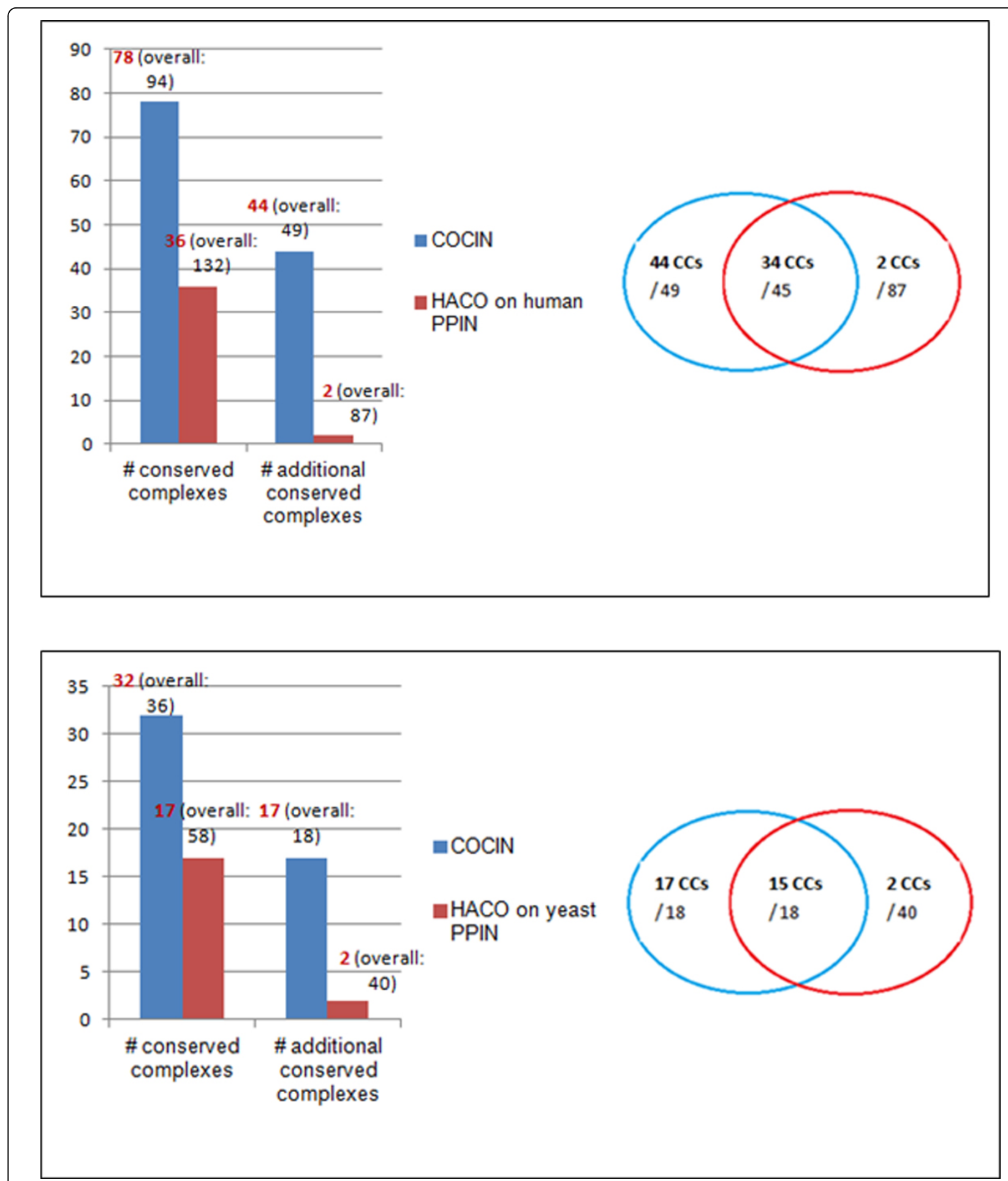

Figure 7 COCIN compared to HACO. COCIN over the interolog network identifies significantly more conserved complexes compared to direct clustering of the original PPI networks using HACO [20].

relationships of conservation between human and yeast complexes.

Sharan et al. used whole-sequence similarity to construct the interolog network. Here, we used OrthoMCL as a substitute for the whole-sequence similarity due to technical difficulties of running BLAST for a large number of proteins. We compared the performance of using OrthoMCL against using Ensembl, which uses domain 

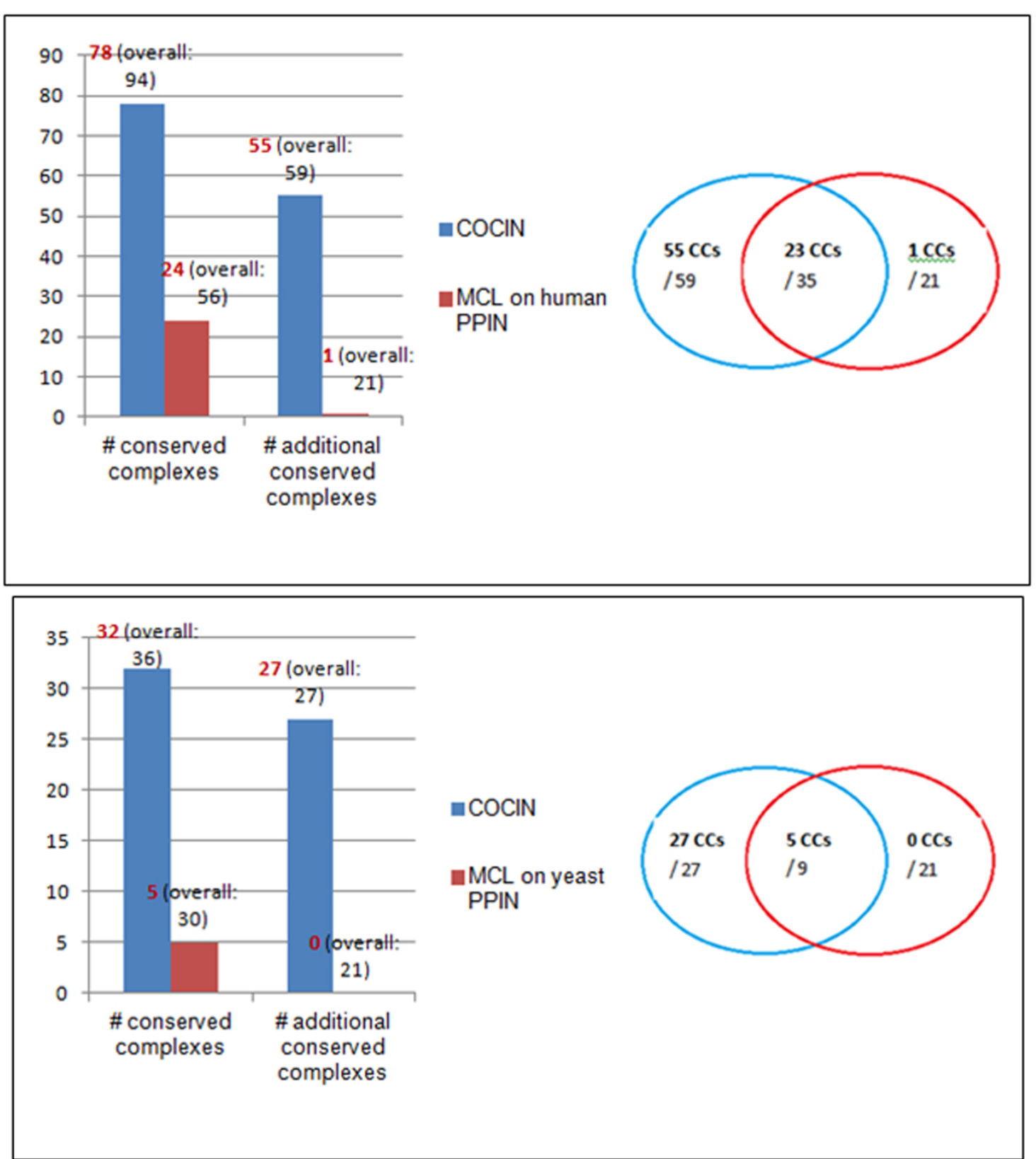

Figure 8 COCIN compared to MCL. COCIN over the interolog network identifies significantly more conserved complexes compared to direct clustering of the original PPI networks using MCL [21].

Table 9 Details of gold standard testing dataset for conserved protein complexes between human and yeast

\begin{tabular}{ll}
\hline Score usage & MSJ $\geq$ threshold \\
\hline Threshold & $50 \%$ \\
\hline \# conserved yeast complexes & $\frac{\mathbf{4 2} / 149 \text { with size }>3(28.1 \%)}{$\cline { 2 - 2 } \text {$Total: 79/408 (19.3\%) }}$ \\
\hline \# conserved Human complexes & $\frac{\mathbf{1 1 8} / 722 \text { with size }>3(16.3 \%)}{\text { Total: 219/1843 (11.9\%) }}$ \\
\hline
\end{tabular}

conservation along with sequence similarity to determine orthology. Table 10 and Figure 11 show that we obtain an overall improvement in terms of the number of mapped protein pairs, interologs, as well as conserved protein complexes in both human and yeast by incorporating domain information (through Ensembl). This substantiates the improved performance of COCIN over traditional sequence-similarity based methods. 

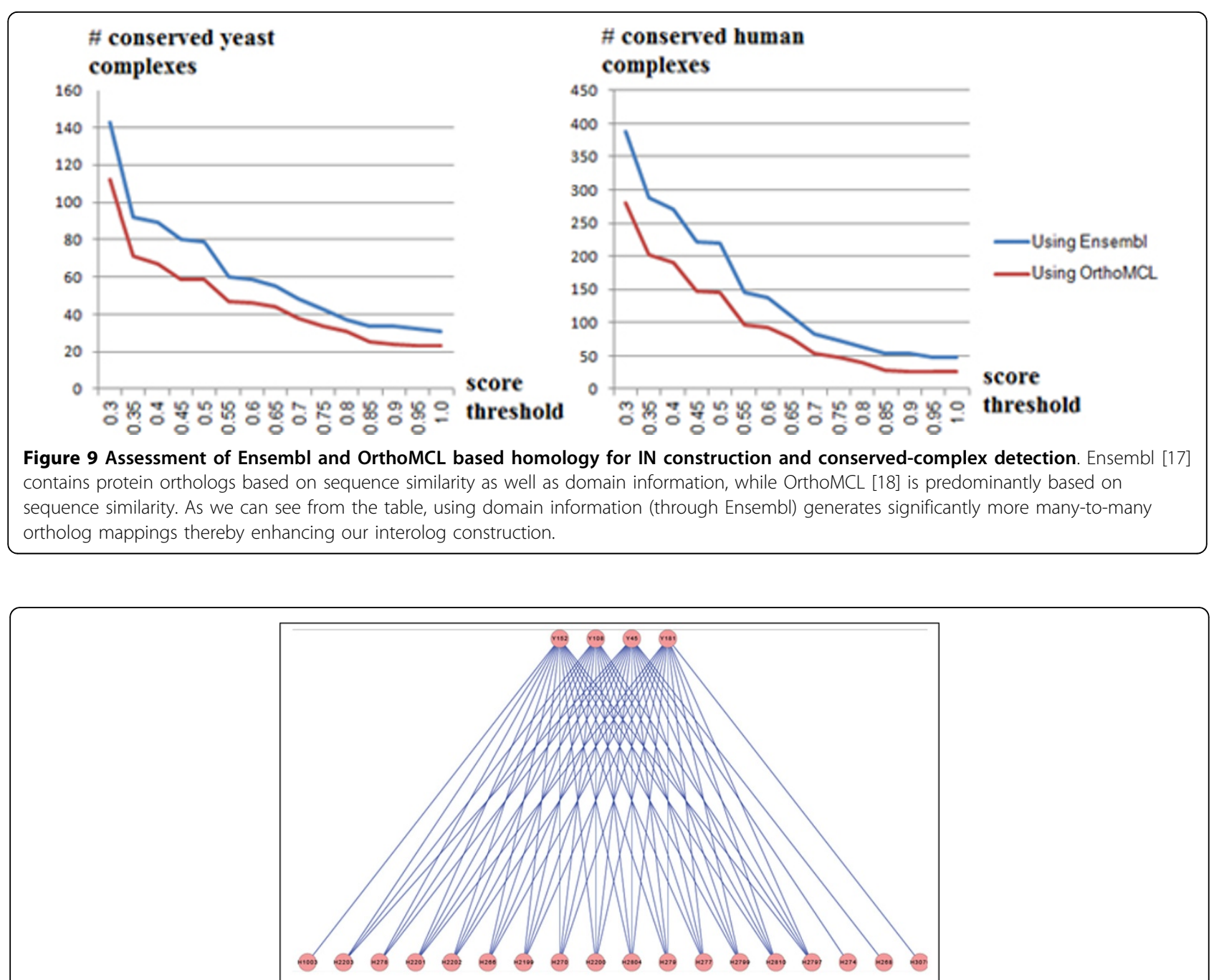

a) Conservation between DNA replication factor $\mathrm{C}$ complex familes of yeast (the above row) and human (the below row).

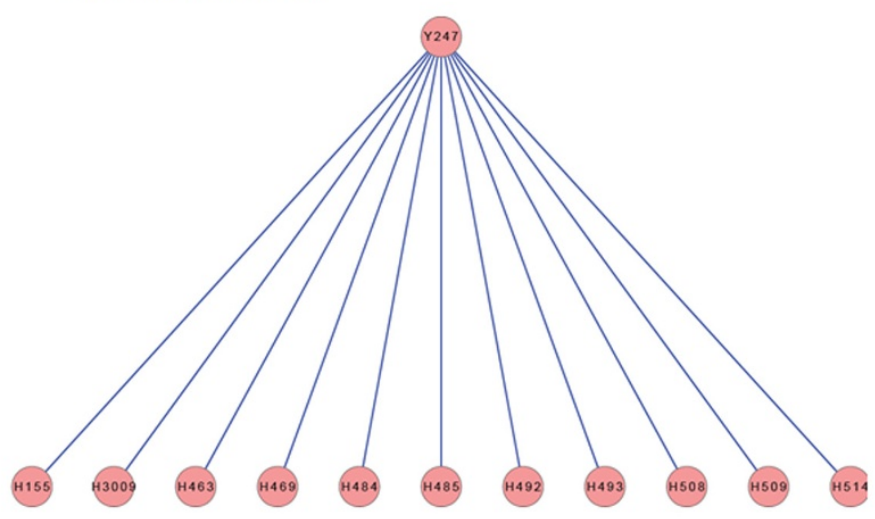

b) Conservation between the transcription factor TFIID complex of yeast and the transcription factor TFIID complex family of human (the below row).

Figure 10 Some examples of the one-to-many and many-to-many relationships of complex conservation between human and yeast Ensembl [17] contains protein orthologs based on sequence similarity as well as domain information, while OrthoMCL [18] is predominantly based on sequence similarity. As we can see from the table, using domain information (through Ensembl) generates significantly more many-tomany ortholog mappings thereby enhancing our interolog construction. 
Table 10 Homology data: Ensembl and OrthoMCL

\begin{tabular}{llll}
\hline & & Ensembl database & OrthoMCL database \\
\hline \# Ortholog groups: & \# 1-to-1 groups & 1096 & 1153 \\
\cline { 2 - 4 } & $\#$ 1-Yeast-to-many groups & 756 & 434 \\
\cline { 2 - 4 } & $\#$ 1-Human-to-many groups & 116 & 116 \\
\cline { 2 - 4 } & \# many-to-many groups & 197 & 167 \\
\cline { 2 - 4 } & Total: & $\mathbf{2 1 6 5}(5503$ pairs) & $\mathbf{1 8 7 0}$ \\
\hline \# Human paralog groups: & & 2573 & 393 \\
\hline \# Yeast paralog groups: & & 426 & 4698 \\
\hline Total \# homolog groups: & & 5164 & \\
\hline
\end{tabular}

Ensembl [17] contains protein orthologs based on sequence similarity as well as domain information, while OrthoMCL [18] is predominantly based on sequence similarity. As we can see from the table, using domain information (through Ensembl) generates significantly more many-to-many ortholog mappings thereby enhancing our interolog construction.

\section{Discussion}

Figure 1 paints a very complicated picture for the conservation pattern of protein complexes from yeast to human. We believe that this picture reflects the actual situation, and it overrides the belief that a yeast complex is essentially a (proper) subset of a human complex with only a few new proteins added to the human complex. In other words, the conservation pattern from yeast to human is highly intricate involving dispersion and re-distribution of proteins and their functions across complexes along with addition of several new proteins in human. At a very high level, though core cellular mechanisms such as cell cycle and DDR are indeed conserved from yeast to human, within these mechanisms, considerable re-arrangements have occurred. This finding can have implications on studies attempting to extrapolate relationships such as synthetic lethality (SL) from yeast to human. In particular, we believe that many of the SL relationships may not be conserved from yeast to human, or even if conserved, may not be identifiable by simple BLAST-based sequence-similarity mappings.

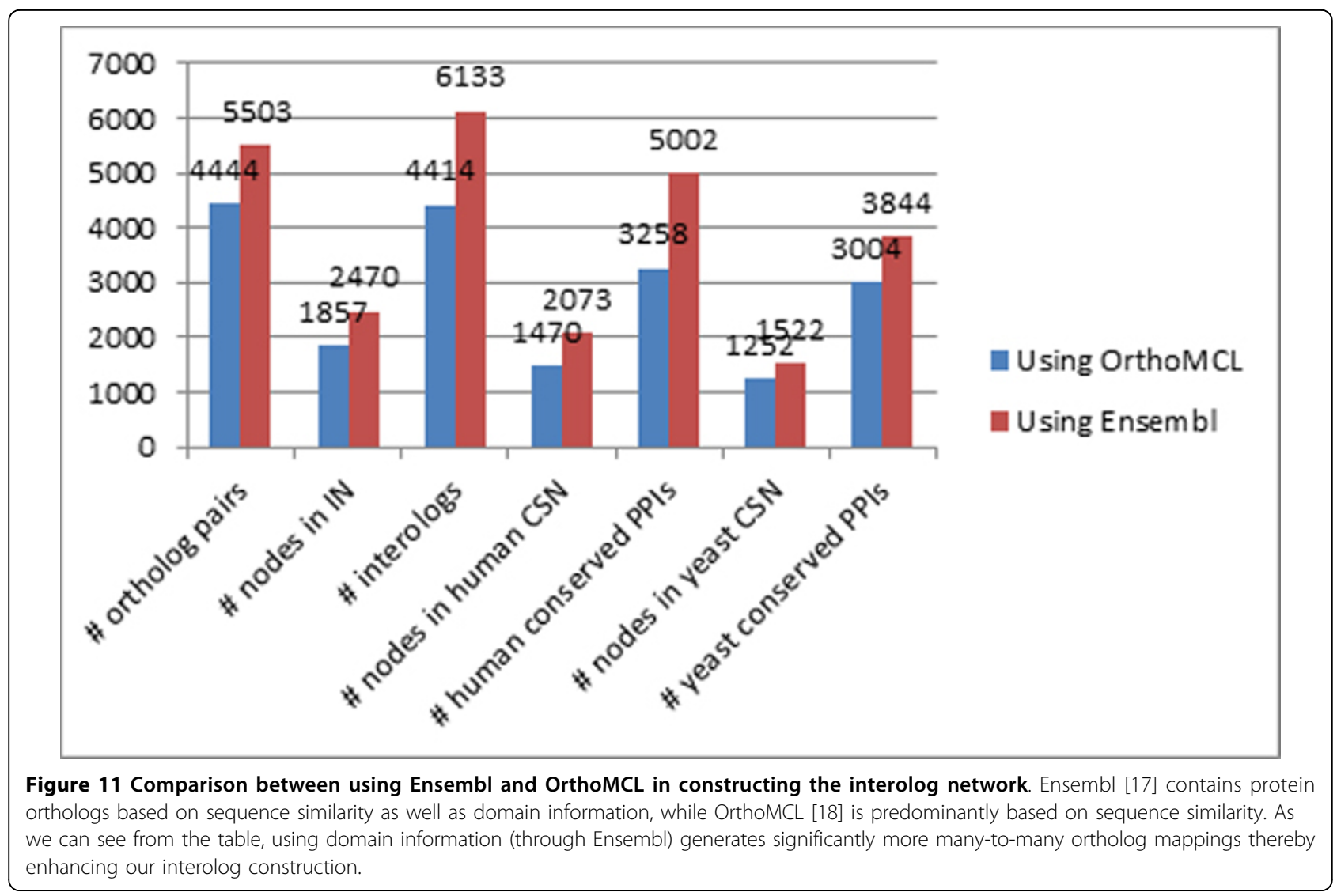




\section{Conclusions}

Identifying conserved complexes between species is a fundamental step towards identification of conserved mechanisms from model organisms to higher level organisms. Current methods based on clustering PPI networks do not work well in identifying conserved complexes, and they are severely limited by lack of true interactions and presence of large amounts of false interactions in existing PPI datasets. Here, we presented a method COCIN based on building interolog networks from the PPI networks of species to identify conserved complexes. Our experiments on yeast and human datasets revealed that our method can identify considerably more conserved complexes that plain clustering of the original PPI networks. Further, we demonstrated that integrating domain information generates many-to-many ortholog relationships which significantly enhances interolog quality and throws further light on conservation of mechanisms between yeast and human.

\section{Availability}

Our COCIN software and the datasets used in this work are freely available at: http://www.comp.nus.edu.sg/ leonghw/ COCIN/ or alternately at: https://sites.google.com/site/ mclcaw/. The preliminary version of this work appeared as a poster paper (abstract) at RECOMB 2013 [30].

\section{Competing interests}

The authors declare that they have no competing interests.

\section{Authors' contributions}

All the authors have equal contributions to the ideas of the work. PVN performed the experiments and analysis, software development. SS and PVN took part in writing the manuscript. HWL supervised the project and reviewed the manuscript. All the authors have read and approved the manuscript.

\section{Acknowledgements \\ PVN wants to thank the RAS Group at School of Computing, National University of Singapore for all the helpful discussions he had during this work; he also thanks Professor Hoai-Bac Le and the University of Science, Vietnam National University at Ho Chi Minh City for the moral support during his overseas study.}

\section{Declarations}

Publication of this article was funded by an NUS Singapore ARF grant R252000-461-112, and SS was supported by an Australian NHMRC grant 1028742 to Dr Peter T. Simpson and Professor Mark A. Ragan.

This article has been published as part of BMC Bioinformatics Volume 14 Supplement 16, 2013: Twelfth International Conference on Bioinformatics (InCoB2013): Bioinformatics. The full contents of the supplement are available online at http://www.biomedcentral.com/bmcbioinformatics/ supplements/14/S16.

\section{Authors' details}

'Department of Computer Science, National University of Singapore, Singapore 117590. ${ }^{2}$ Institute for Molecular Bioscience, The University of Queensland, St. Lucia, QLD 4072, Australia.

Published: 22 October 2013
References

1. Srihari S, Leong HW: A survey of computational methods for protein complex prediction from protein interaction networks. Journal of Bioinformatics and Computational Biology 2013, 11(2):1230002.

2. Li X, Min Wu, Ng SK: Computational approaches for detecting protein complexes from protein interaction networks: a survey. BMC Genomics 2010, 11(Suppl 1):S3.

3. Srihari S, Ragan MA: Systematic tracking of dysregulated modules identifies novel genes in cancer. Bioinformatics 2013, 29(12):1553-1561.

4. Vanunu O, Magger O, Ruppin E, Shlomi T, Sharan R: Associating genes and protein complexes with disease via network propagation. PLOS Computational Biology 2010, 6(1):e1000641.

5. Havugimana PC, Hart GT, Nepusz T, Yang H, Turinsky AL, Li Z, Wang PI, Boultz DR, Fong V, Phanse S, Babu M, Craig SA, Hu P, Wan C, Vlashblom J, Dar VU, Bezginov A, Clark GW, Wu GC, Wodak SJ, Tillier ER, Paccanaro A, Marcotte EM, Emili A: A consensus of human soluble protein complexes. Cell 2012, 150(5):1068-1081.

6. Khanna KK, Jackson SP: DNA double-strand breaks: signalling, repair and the cancer connection. Nature Genetics 2001, 27:247-254.

7. Xu B, Seong-tae K, Kastan MB: Involvement of BRCA1 in S-phase and G2phase checkpoints after ionizing irradiation. Molecular Cell Biology 2001, 21:3445-3450.

8. Wang Y, Cortez D, Yazdi P, Neff N, Elledge SJ, Qin J: BASC, a super complex of BRCA1-associated proteins involved in the recognition and repair of aberrant DNA structures. Genes and Development 2000, 14:927-939.

9. Kelley BP, Sharan R, Karp RM, Sittler T, Root DE, Stockwell BR, Ideker T: Conserved pathways within bacteria and yeast as revealed by global protein network alignment. Proceedings of the National Academy of Sciences USA 2003, 100:11394-11399.

10. Sharan R, Ideker T, Kelley B, Shamir R: Identification of protein complexes by comparative analysis of yeast and bacterial protein interaction data. Journal of Computational Biology 2005, 12(6):835-846.

11. van Dam JP, Snel B: Protein complex evolution does not involve extensive network rewiring. PLoS Computational Biology 2008, 4(7):e1000132.

12. Hirsh E, Sharan R: Identification of conserved protein complexes based on a model of protein network evolution. Bioinformatics 2007, 23(2): e170-e176.

13. Zhenping L, Zhang S, Wang Y, Zhang XS, Chen L: Alignment of molecular networks by integer quadratic programming. Bioinformatics 2007, 23(13):1631-1639.

14. Marsh JA, Hernandenz H, Hall Z, Ahnhert SE, Perica T, Robinson CV, Teichmann SA: Protein complexes are under evolutionary selection to assemble via ordered pathways. Cell 2011, 153(2):461-470.

15. Bork P, Hoffman K, Bucher P, Neuwald AF, Alstchul SF, Koonin EV: A superfamily of conserved domains in DNA damage-responsive cell cycle checkpoint proteins. FASEB Journal 1997, 11(1):68-76.

16. Larsen NB, Hickson ID: RecQ helicases: conserved gaurdians of genomic integrity. DNA Helicases and DNA Motor Proteins: Advances in Experimental Medicine and Biology (Springer New York) 2013, 973:161-184.

17. Flicek P, Amode MR, Barrell D, Beal K, Brent S, Carvalho-Silva D, Clapham P, Coates G, Fairley S, Fitzgerald S, Gil L, Gordon L, Hendrix M, Hourlier T, Johnson N, Kähäri AK, Keefe D, Keenan S, Kinsella R, Komorowska M, Koscielny G, Kulesha E, Larsson P, Longden I, McLaren W, Muffato M, Overduin B, Pignatelli M, Pritchard B, Riat HS, Ritchie GR, Ruffier M, Schuster M, Sobral D, Tang YA, Taylor K, Trevanion S, Vandrovcova J, White S, Wilson M, Wilder SP, Aken BL, Birney E, Cunningham F, Dunham I, Durbin R, Fernández-Suarez XM, Harrow J, Herrero J, Hubbard TJ, Parker A, Proctor G, Spudich G, Vogel J, Yates A, Zadissa A, Searle SM: Ensembl 2012. Nucleic Acids Research 2012, 40:D84-D90.

18. Li L, Stoeckert CJ, Ross DS: OrthoMCL: Identification of ortholog groups for eukaryotic genomes. Genome Research 2003, 13:2178-2189.

19. Liu G, Wong L, Chua HN: Complex discovery from weighted PPI networks. Bioinformatics 2009, 25(15):1891-1897.

20. van Dongen SM: Graph clustering by flow simulation. PhD thesis University of Utrecht; 2000

21. Wang H, Kakaradov B, Collins SR, Karotki L, Fiedler D, Shales M, Shokat KM, Walther TC, Krogan NJ, Koller D: A complex-based reconstruction of the Saccharomyces cerevisiae interactome. Molecular and Cellular Proteomics 2009, 8(6):1361-1381. 
22. Srihari S, Leong HW: MCL-CAw: a refinement of $M C L$ for detecting yeast complexes from weighted PPI networks by incorporating coreattachment structure. BMC Bioinformatics 2010, 11:504.

23. Srihari S, Leong HW: Temporal dynamics of protein complexes in PPI networks: a case study using yeast cell cycle dynamics. BMC Bioinformatics 2012, 17(S16).

24. Srihari S, Leong HW: Employing functional interactions for characterization and detection of sparse complexes from yeast PPI networks. International Journal of Bioinformatics Research and Applications 2012, 8:286-304, Nos. 3/4, September.

25. Kerrien S, Alam-Faruque Y, Aranda B, Bancarz I, Bridge A, Derow C, Dimmer E, Feuermann M, Friedrichsen A, Huntley R, Kohler C, Khadake J, Leroy C, Liban A, Lieftink C, Montecchi-Palazzi L, Orchard S, Risse J, Robbe K, Roechert B, Thorneycroft D, Zhang Y, Apweiler R, Hermjakob H: IntAct - open source resource for molecular interaction data. Nucleic Acids Research 2007, 35:D561-D565.

26. Stark C, Breitkreutz BJ, Chatr-Aryamontri A, Boucher L, Oughtred R, Livstone MS, Nixon J, Van Auken K, Wang X, Shi X, Reguly T, Rust JM, Winter A, Dolinski K, Tyers M: The BioGrid Interaction Database: 2011 Update. Nucleic Acids Research 2011, 39(Suppl 1):D698-D704.

27. Keshava Prasad TS, Goel R, Kandasamy K, Keerthikumar S, Kumar S, Mathivanan S, Telikicherla D, Raju R, Shafreen B, Venugopal A, Balakrishnan L, Marimuthu A, Banerjee S, Somanathan DS, Sebastian A, Rani S, Ray S, Harrys Kishore CJ, Kanth S, Ahmed M, Kashyap MK, Mohmood R, Ramachandra YL, Krishna V, Rahiman BA, Mohan S, Ranganathan P, Ramabadran S, Chaerkady R, Pandey A: Human Protein Reference Database - 2009 Update. Nucleic Acids Research 2009, 37: D767-D772.

28. Pu S, Wong J, Turner B, Cho E, Wodak SJ: Up-to-date catalogue of yeast protein complexes. Nucleic Acids Research 2009, 37(3):825-831.

29. Ruepp A, Waegele B, Lechner M, Brauner B, Dunger-Kaltenbach I, Fobo G, Frishman G, Montrone C, Mewes HW: CORUM: the comprehensive resource of mammalian protein complexes. Nucleic Acids Research 2008, 36(Database):D646-650.

30. Nguyen PV, Srihari S, Leong HW: Identifying conserved protein complexes between species by constructing interolog interaction networks, (poster paper). 17th International Conference on Research in Computational Molecular Biology (RECOMB 2013) 2013, April.

doi:10.1186/1471-2105-14-S16-S8

Cite this article as: Nguyen et al.: Identifying conserved protein complexes between species by constructing interolog networks. BMC Bioinformatics 2013 14(Suppl 16):S8.

\section{Submit your next manuscript to BioMed Central and take full advantage of:}

- Convenient online submission

- Thorough peer review

- No space constraints or color figure charges

- Immediate publication on acceptance

- Inclusion in PubMed, CAS, Scopus and Google Scholar

- Research which is freely available for redistribution

Submit your manuscript at www.biomedcentral.com/submit
Biomed Central 